\title{
Poor dietary practice and its associated factors among patients with type 2 diabetes mellitus on follow up in Nigist Elleni Mohammed Memorial Comprehensive Specialized Hospital, Ethiopia
}

Mulugeta Selassie

Hadiya Zone health department

Desalegn Tamiru

Jima University

Tegegn Tadesse ( $\nabla$ tegegntadesse24@gmail.com )

Wachamo University

Adisalem Gizachew

Wachamo University

\section{Research Article}

Keywords: Adherence, Type 2, Diabetes mellitus, diet, Ethiopia

Posted Date: August 30th, 2021

DOI: https://doi.org/10.21203/rs.3.rs-819197/v1

License: (c) (1) This work is licensed under a Creative Commons Attribution 4.0 International License.

Read Full License 


\section{Abstract \\ Background}

Despite the importance of dietary practice on self care of patients with type 2 diabetes mellitus, poor dietary practice results in long term complications. Therefore this study was conducted to identify the level of poor dietary practice among type 2 diabetic patients on follow up in Nigist Elleni Mohammed Memorial comprehensive specialized Hospital, Southern Ethiopia.

\section{Methods}

Facility based cross-sectional study design was employed to assess the level of poor dietary practice among type II diabetes mellitus adult patients. The study was conducted from March to April 2020 G.C. Systematic random sampling method was used to select the study respondent. Both bivariate and multivariable logistic regressions were carried out to assess independent predictors of poor practice to diet. Odds ratios and their $95 \%$ confidence intervals together with value $\leq 0.05$ were used to identify independent predictors of poor dietary practice.

\section{Result}

The overall proportion of poor dietary adherence among type diabetes patients was $53.7 \%(n=168)$. Having large family size, occupations, being low wealth status, lack of family support and not being member of diabetic association were the factors associated with poor adherence of dietary practice among type 2 diabetes mellitus patients.

\section{Conclusion}

The overall magnitude of the poor adherence to dietary practice among type 2 diabetes mellitus adult patients was $53.7 \%$ which is not going in line with international recommendations of diabetic self care. Every concerned body should strive to address those factors associated with poor dietary practice among type 2 diabetes mellitus patients.

\section{Background}

Diabetes mellitus is a clinical syndrome characterized by hyperglycemia due to absolute or relative deficiency of insulin. It is clinically categorized DM as Type I diabetes, Type II diabetes, Gestational diabetes mellitus and other specific types of diabetes due to other causes such as genetic defects in bcell function, genetic defects in insulin action and diseases of the exocrine pancreas [1]. 
The international diabetes federation estimates that 463 million adults are living with diabetes worldwide with 1 in 11 people are living with diabetes. Worldwide Diabetes Mellitus (DM) is one of the most challenging health problems. Ongoing patient self-management, education, and support are critical to prevent acute complications and to reduce the risk of long-term complications from the disease. Also adherence to recommended meal plans/dietary schemes and being active can keep blood glucose level, blood pressure, and cholesterol levels within optimum ranges. It follows that non-adherence to recommended diet would lead to life-threatening complications in individuals with diabetes [2].

Recommended dietary practices or dietary adherence include consuming more fruits and vegetables, nuts and whole grains, and choosing unsaturated vegetable oil as opposed to saturated animal based fats. It also includes limiting consumption of snacks that are high in fat, sugar, or salt [3].

Patients need to achieve and maintain a healthy body weight, perform a regular physical activity for at least $30 \mathrm{~min}$ and moderate-intensity activity on most days, eat a healthy diet, and avoid sugar and saturated fats intake and tobacco use in order to prevent type2 DM and its complication [2].

Even though dietary modification is one of the corner stone in T2 DM management and is usually recommended as the first step, it is considered as one of the most challenging aspects of diabetes management. Implementation of recommended dietary practice for individual's with T2 DM requires collaboration between the patient and the healthcare provider [4].

Non adherence to dietary recommendation in people with T2 DM has been identified as high in both developed and developing countries [5]. None adherence to recommended dietary recommendation in diabetes mellitus patients may results in long term complications [6]. Some of the factors associated with poor adherence to dietary recommendations are socioeconomic status, duration of illness, duration of follow up, educational level, coo morbidity, family support, lack of diabetes knowledge, cost of healthy diet and poor communication with healthcare providers are among the most common factors [7]. But there is, limited information regarding the level of adherence and factors associated with poor dietary recommendations in individuals with T2DM in Africa including Ethiopia. A few reports in Ethiopia and other parts of Africa suggest that adherence to dietary recommendation in DM patients are generally low [8].

In Ethiopia, lack of information on adherence to dietary recommendation and absence of dietary practice guideline for people withT2DM is still challenging. Moreover, due to little evidence-based researches were done, the health policy of Ethiopia is still unable to give evidence based decision. Therefore conducting and documenting such research would have a positive impact on designing and implementation of dietary practice programs for people with T2DM in Ethiopia.

There is a limited literature regarding dietary adherence among type II diabetes patients who are on follow up in public health care in Ethiopia, particularly in the study area, therefore this study was conducted to identify the level of adherence to dietary recommendation among type 2 diabetic patients on follow up in Nigist Elleni Mohammed Memorial comprehensive specialized Hospital, southern Ethiopia. 


\section{Objectives}

\section{General objective}

- To assess adherence to dietary recommendation and associated factors among type 2 diabetes mellitus patients on follow up in Nigist Elleni Mohammed Memorial Referral and Teaching Hospital, Southern Ethiopia, 2020

\section{Specific objective}

- To verify the level of adherence to dietary recommendation among type 2 diabetic patients on follow up

- To identify factors associated with adherence to dietary recommendation among type 2 DM patients on follow up

\section{Methods And Materials}

\section{Study setting}

The study was conducted Nigist Eleni Mohammed Memorial Referral and teaching Hospital which is located in Hadiya zone in South Nation's Nationalities and Peoples Region found in $232 \mathrm{kms}$ south of Addis Ababa, Ethiopia. Currently it provides preventive, curative and rehabilitative clinical services organized in four case teams as outpatient, inpatient, emergency and critical care, maternal, child health and obstetrics and operation theatre. The out patients services are given in OPD clinics (internal medicine, surgery, pediatrics and child health, Gynecologic), specialty clinics (psychiatry, dermatology, ophthalmology, Dentistry and Orthopedic), referral and consultation clinics, Maternal and child health care follow up clinics.

The study was carried out in Nigest Eleni Mohammed Memorial Referral and Teaching Hospital, Southern Ethiopia, from March to April 2020 G.C [9].

\section{Study design}

Facility based cross-sectional study was employed to assess the level of adherence to dietary recommendations among type II diabetes mellitus adult patients.

\section{Source population}

The study source population was all patients with type 2 diabetes aged 18 years and above who were on diabetic follow up at Nigist Elleni Mohammed Memorial Teaching and Referral Hospital.

\section{Study Population}


The study population was Type 2 diabetic patients aged18 years and above who were visiting Nigist Elleni Mohammed Memorial Teaching and Referral Hospital diabetes clinic and randomly selected for the study

\section{Inclusion Criteria}

Patients those who were diagnosed with type 2 diabetes and on clinical care for at least one year were included.

\section{Exclusion Criteria}

A patient who was critically ill and need immediate treatment during data collection period was excluded from the study

\section{Sample size determination}

The sample size for the study was determined using the single population proportion formula for sample size determination [10].

$$
\frac{(\mathrm{Z} \alpha / 2) 2 * \mathrm{P} *(1-\mathrm{p})}{(\mathrm{d}) 2}
$$

$$
\begin{aligned}
& =\frac{(1.96) 2 * 0.743 *(1-0.743)}{(0.05) 2} \\
& =\frac{3.8416 * 0.743 *(0.257)}{(0.0025)}=\frac{0.73356}{(0.0025)}=293
\end{aligned}
$$

By considering non-response rate of $10 \%$ the final sample size was 322.

\section{Sampling techniques}

Nigist Eleni Referral Hospital is selected purposively since it is the only hospital in the town. Systematic random sampling method was used to select the study respondent. There were 862 type II diabetes mellitus patients on follow up age of 18 and greater at the time. Each patient visits the facility on monthly basis. To run systematic sampling the K-value (the interval) was calculated by dividing total number of type 2 DM patients (862) from diabetes referral clinic registration log book to the calculated sample size (322). Therefore $\mathrm{K}=\mathrm{N} / \mathrm{nf}$, where $\mathrm{nf}=$ final sample size $=322$ and $\mathrm{N}=$ total Number of type 2 diabetes patients who are attending the hospital for follow up, which is equal to 862 patients. Accordingly, every $2^{\text {nd }}$ patients were selected until the sample size fills with the first sample chosen randomly between 1 and $\mathrm{k}$.

\section{Study Variables}

\section{Dependent variable}


Adherence to dietary recommendation

\section{Independent variable}

Socio demographic characteristics: Age, Sex, religion, marital status, type of work, income, educational status.

Disease related: duration of illness, duration of follow up, other co morbidity, family support, attending dietary education.

Food related: cost of the food, availability of the food, type of food that should be consumed, frequency and meal timings, member of diabetic association.

Knowledge about the recommended diet

\section{Data collection instrument and personnel}

Data was collected using a structured interviewer administered questionnaire. The questionnaire was initially prepared in English then translated in to local language (Amharic) by professionals who were fluent two languages and then translated back to English to ensure consistency. In order to ensure reliability and consistency, enumerators were trained for 1 day. Pretest was undertaken by considering $5 \%$ of the sample size in Homacho hospital located outside the study area. Findings of the pretest were incorporated to modify and clarify the collection tool before the actual data collection. Data were collected by four trained nurses and two supervisors for five consecutive weeks from March to April 2020 G.C. The data collection tool used in this study was adapted and modified from previous studies on similar topics.

To determine the dietary non-adherence of individuals with DM, we used a modified form of the eight items Morisky medication adherence scale (MMAS-8) which was modified by Worku et al. [7]. This scale has 11 components and was computed by taking the mean value to classify the respondents' poor dietary practice as "dietary non-adherence" and good practice as "dietary adherence". The Perceived Dietary Adherence Questionnaire (PDAQ) was used for dietary adherence measurement. Each of the items contain two response options ( $\mathrm{Yes}=1$ and $\mathrm{No}=0$, here yes was used for those responses which are negatively answered or far true answer from what science is talking about). The questionnaire was tested for internal consistency (reliability) with Cronbach's Alpha test (0.7). The completeness, consistency, and accuracy of the collected data were examined by principal investigators every day.

\section{Data processing and analysis}

Data coding was done at the end of each day of data collection and recoded later where necessary. Data were entered and analyzed by using SPSS version-20 statistical package. House hold wealth index was determined from asset data using principal component analysis (PCA). First, variables were coded between 0 and 1 , and then the variables entered and analyzed using PCA and those variables which have 
commonality values greater than 0.5 were used to produce factor scores. Frequency distributions, percentages, tables and charts were used to show results of univariate analysis. Bivariate and multivariable logistic regressions were carried out to assess independent predictors of non adherence to diet. Bivariate logistic regression was performed to identify candidate variables for multivariable logistic regression. Variables with value $\leq 0.25$ in bivariate regression were considered as candidates for multivariable regression. Odds ratios and their $95 \%$ confidence intervals together with value $\leq 0.05$ were used to identify independent predictors of non adherence to diet. Goodness of fitness of the final model was checked using Hosmer and Lemeshow adequacy of model test.

\section{Operational definitions}

Poor dietary adherence: respondents who answered incorrectly on 10 items of perceived dietary adherence questions and those who scored above the mean value were classified as poor adherence to dietary recommendation [10].

Good dietary adherence: respondents who answered correctly on 10 items of perceived dietary adherence questions and those who scored equal or below the mean value were classified as good adherence to dietary recommendation [10].

Good Dietary Knowledge: respondents who answered correctly to knowledge related questions and those who scored greater than the overall mean value.

Poor Dietary Knowledge: respondents who answered in-correctly to knowledge related questions and those who scored less than or equal to overall mean value.

\section{Ethical consideration}

Ethical clearance was obtained from ethical review board of wachemo university department of public health research ethical committee. Official letter of permissions was obtained from Nigist Eleni Mohammed Memorial hospital medical director office and respondents were well informed about the purpose of the study, then data was collected after written consent from each participant obtained. Information was recorded anonymously and confidentiality and beneficence was assured throughout the study period.

\section{Results}

\section{Socio demographic characteristics of the respondents}

A total of 313 respondents agreed to participate in the study and $97.2 \%$ of the participants gave complete response. More than half of the participants $(56.9 \%)$ were males. The mean age ( \pm S.D) of type $2 \mathrm{DM}$ patients was $48.12( \pm 11.348)$ years with the age ranges between $20-90$ years. The majority of the participants $187(59.7 \%)$ were between 41 and 60 years. Among the respondents, majority of them 214 and $240(68.4 \%$ and $76.7 \%)$ were from protestant Christian followers and from Hadiya ethnic group 
respectively. Regarding to educational status, $51(16.3 \%)$ and $99(31.6 \%)$ of the patients attended higher education and had no formal education respectively. Of the total study participants, $116(37.1 \%)$ of the respondents were rural residents .Nearly half of respondents $148(47.3 \%)$ had $4-6$ family size and $83(26.5 \%)$ of the respondents were government employees. More than one third (34.8\%) of the study participants were from low or poor economic status (Table 1).

Table 1: Socio-demographic characteristics of type 2 diabetic adult patients at Nigist Elleni Mohammed Memorial Referral and Teaching Hospital, Southern Ethiopia, $2020(n=313)$. 


\begin{tabular}{|c|c|c|c|}
\hline \multirow[t]{3}{*}{ Sex } & Female & 135 & 43.1 \\
\hline & Male & 178 & 56.9 \\
\hline & Less than 40years & 87 & 27.8 \\
\hline \multirow[t]{3}{*}{ Age in years } & $41-60$ years & 187 & 59.7 \\
\hline & $>60$ years & 39 & 12.5 \\
\hline & Less than 3 & 49 & 15.7 \\
\hline \multirow[t]{3}{*}{ Family size } & $4-6$ & 148 & 47.3 \\
\hline & Greater than 6 & 116 & 37 \\
\hline & Protestant & 214 & 68.4 \\
\hline \multirow[t]{3}{*}{ Religion } & Orthodox & 57 & 18.2 \\
\hline & Muslim & 30 & 9.6 \\
\hline & Catholic & 11 & 3.5 \\
\hline \multirow[t]{3}{*}{ Marital status } & Married & 297 & 94.9 \\
\hline & Single & 16 & 5.1 \\
\hline & Urban & 197 & 62.9 \\
\hline \multirow[t]{2}{*}{ Residence } & Rural & 116 & 37.1 \\
\hline & Hadiya & 240 & 76.7 \\
\hline \multirow[t]{5}{*}{ Ethnicity } & Kembata & 25 & 8 \\
\hline & Gurage & 13 & 4.2 \\
\hline & Amahara & 22 & 7 \\
\hline & Silitie & 13 & 4.2 \\
\hline & Unable to read and write & 99 & 31.6 \\
\hline \multirow[t]{5}{*}{ Educational status } & Able to read and write & 48 & 15.3 \\
\hline & Primary school & 51 & 16.3 \\
\hline & Secondary school & 64 & 20.4 \\
\hline & Higher education & 51 & 16.3 \\
\hline & Farmer & 69 & 22 \\
\hline
\end{tabular}




\begin{tabular}{llcl}
\multirow{2}{*}{ Variable } & Categories & Frequency(n) & Percentage \\
\hline \multirow{2}{*}{ Occupation } & Government employee & 83 & 26.5 \\
\cline { 2 - 4 } & Merchant & 71 & 22.7 \\
\cline { 2 - 4 } & Private organization employee & 6 & 1.9 \\
\hline Daily laborer & 5 & 1.6 \\
\hline House wife & 73 & 23.3 \\
\hline \multirow{2}{*}{ Wealth } & Other & 6 & 2 \\
\cline { 2 - 3 } & Low & 109 & 34.8 \\
& Medium & 104 & 33.2 \\
\hline
\end{tabular}

\section{Health related characteristics}

The mean duration of patients follow up was 6.12(SD \pm 4.575$)$ years, which is ranging from 1 to 5 years $(51.1 \%)$ to greater than 10 years $(11.8 \%)$. More than one third of participants $(35.8 \%)$ had chronic disease other than DM, mainly hypertension $83(26.5 \%)$ followed by heart disease $15(4.8 \%)$. One in four patients $(21.7 \%)$ had family history of DM. Only $11.2 \% \& 1.3 \%$ of patients control their DM condition by diet and exercise respectively. A large number of participants (93.9\%) made change in dietary habit when they knew that they were diabetic. Significant percentage (41.9\%) of the study participants had participated in regular physical exercise for less than 3 times per a week for more than 30 minutes. More than one in three of the study participants (36.1\%) indicated that family support affects their dietary habits. A large number of study participants $(97.1 \%)$ attended education on dietary recommendation necessary for management of DM by doctors $(61.3 \%)$ as well as by nurses $(30.7 \%)$.

From the total study participants more than half of the study participants $171(54.6 \%)$ had poor dietary knowledge. Among those who had poor dietary knowledge male accounts $30.6 \%$. More than half of the study participants (53\%) were practicing dietary modification to control DM complication while $32.9 \%$ of them were using exercise as life style modification to control DM complication. Majority of the study participants $(82.1 \%)$ responded fruit and vegetables as food groups which they used to control their blood glucose level.

\section{Level of dietary adherence among Type II adult patients}

The overall proportion of poor dietary adherence among type diabetes patients was $168(53.7 \%)[95 \% \mathrm{Cl}$ $(48.2,58.8)]$. The proportion of poor dietary adherence among male respondents was $55.4 \%[95 \% \mathrm{Cl}(52$, 
62.9)] .According to this study the proportion of poor dietary adherence practice among age groups of less than 40 years was $28.6 \%$ [ $95 \% \mathrm{Cl},(22,32.6)]$ and it was $10.7 \%$ [ $95 \% \mathrm{Cl},(8.9,16.3)]$ among the age groups of 60 years and above.

More than half of the respondents $(57.8 \%)$ reported that they were an unable to follow dietary recommendation due to unavailability of food items, cost of foods, lack of supports from their families and friends and lack of information. A significant number of the study participants (45\%) didn't continue with dietary plan when they felt that their DM is under control. A large number of the study participants $(81.5 \%)$ forgot to consume fruits 3 times or more a day or 6 times or more a week. A large number of patients $(80.5 \%$ \& $78.3 \%)$ responded that they forgot to include fruits and vegetables in their daily foods respectively. More than 3 in 4 patients (76\%) failed to cut down fat intake from their daily foods

\section{Factors associated with poor dietary adherence among type II diabetes mellitus adult patients}

Family size, residence, educational status, occupation, wealth status ,knowledge status of the respondents ,duration of follow up , co- morbidity, fasting blood sugar level, physical exercise ,being member of diabetic association, missed dietary planning, cost of foods, access to fruits and vegetables and family and friends support were selected as candidate for multivariable logistic regression. However in multivariable analysis family size, occupation, wealth status, family and friends support, not being member of diabetic association and missed dietary planning were independently associated with poor dietary adherence among type II DM adult patients (Table 2).

Table 2: Factors associated with dietary adherence in type 2 diabetic patients on follow up at Nigist Elleni Mohammed Memorial Referral and Teaching Hospital, Hossana, SNNPR, Ethiopia ,2020 ( $n=313)$ 
Adherence

Variable

Poor Good

$\mathrm{COR}$
$[95 \% \mathrm{Cl}]$

P- $\quad$ AOR $[95 \% \mathrm{Cl}]$

P-

value

\section{Educational status}

Unable to read \& write

$\begin{array}{ll}62 & 37\end{array}$

$2.209(1.111$

4.393)

value

\begin{tabular}{l} 
Able to read \& write \\
Primary school \\
Secondary school \\
Higher education \\
\hline Occupation
\end{tabular}

Farmer

$37 \quad 33$

2.404 (1.069-

$5.406)$

$30 \quad 21$

1.883 (0.858-

4.133)

$23 \quad 41$

$41 \quad 0.739(0.348-\quad 0.432$

1.571)

0.024

1.165(

0.453-2.991)

0.872

$22 \quad 29$

1

$0.034 *$

$1.306(0.487$

0.725

$-3.506)$

$0.115^{\star}$

$1.003(0.395$

0.969

$-2.550)$

$0.544(0.231$ -

1.281

0.283

1

Farmer

Government employee

$38 \quad 52$

0.595(0.305-

1.160)

0.128 *

$0.304(0.112$

$0.019 *$

$-0.824)$

$\begin{array}{llll}0.388(0.206- & 0.003^{*} & 0.757(0.224 & 0.655 \\ 0.730) & & -2.561)\end{array}$

Mar chant

$44 \quad 34$

0.687(
$1.319)$

$0.578(0.215$

$-1.558)$

0.279

Others***

49

26

1

1

\section{Family size}

Less than 3

$23 \quad 26$

0.561(0. 286- $0.092^{*}$

0.358 (0.159-

$0.011^{\star *}$

1.100)

$0.806)$

4-6

$74 \quad 74$

0.634 ( 0387

1.038)

$0.598(0.338-$

0.291

1.059)

Greater than 7

71

45

1

1

1

Wealth

Low

68

41

2.949(1.679- $\quad 0.000$

$5.177)$

$1.829(0.828-$

4.041)

0.136

Medium

$64 \quad 40$

2.844 ( $1.612-$

$5.020)$

3.337(1.502-

7.415)

High

$36 \quad 64$

1

1

\section{Residence}

Rural

$73 \quad 43$

$0.012^{*}$

1.719(

0.736-4.014)

0.211 
Adherence

\begin{tabular}{lccllll} 
Variable & Poor & Good & $\begin{array}{l}\mathrm{COR} \\
{[95 \% \mathrm{Cl}]}\end{array}$ & $\begin{array}{l}\text { P- } \\
\text { value }\end{array}$ & AOR [95\%Cl] & $\begin{array}{l}\text { P- } \\
\text { value }\end{array}$ \\
\hline Urban & 95 & 102 & $\begin{array}{l}1.83(1.140- \\
2.914)\end{array}$ & & 1
\end{tabular}

\section{Physical exercise}

\begin{tabular}{|c|c|c|c|c|c|c|}
\hline $\begin{array}{l}3 \text { times a week for } 30 \\
\text { minutes }\end{array}$ & 14 & 28 & $\begin{array}{l}0.349(0.169- \\
0.721)\end{array}$ & $0.004^{*}$ & $\begin{array}{l}0.448(0.158 \\
-1.271)\end{array}$ & 0.131 \\
\hline $\begin{array}{l}\text { Less than } 3 \text { times a week } \\
\text { for } 30 \text { minutes }\end{array}$ & 71 & 59 & $\begin{array}{l}0.841(0.520- \\
1.361)\end{array}$ & 0.481 & $\begin{array}{l}1.197(0.577- \\
2.483)\end{array}$ & 0.630 \\
\hline No physical activity & 83 & 58 & 1 & & 1 & \\
\hline \multicolumn{7}{|c|}{ Access of fruits and vegetables } \\
\hline Yes & 137 & 96 & $\begin{array}{l}2.256(1.341 \\
-3.794)\end{array}$ & $0.002^{*}$ & $\begin{array}{l}1.673( \\
0.726-3.856)\end{array}$ & 0.227 \\
\hline No & 31 & 49 & 1 & & 1 & \\
\hline \multicolumn{7}{|l|}{ High cost of foods } \\
\hline Yes & 138 & 95 & $\begin{array}{l}2.421(1.436 \\
-4.083)\end{array}$ & $0.001^{*}$ & $\begin{array}{l}0.648(0.263- \\
1.597)\end{array}$ & 0.346 \\
\hline No & 30 & 50 & 1 & & 1 & \\
\hline
\end{tabular}

\section{Family support}

Yes

No

\section{Current blood sugar level}

Less than $126 \mathrm{mmHg}$

28

129

60

$39 \quad 85$

1

$4.686(2.878-$
$7.629)$

1
4.842( 2.376-9.868)
$0.000^{* *}$

Greater than $126 \mathrm{mmHg}$

$127 \quad 99$

$\begin{array}{ll}43 & 0.650(0.405- \\ 1.042)\end{array}$
0.074 *
$0.596(0.277-$
$1.284)$
0.186

Duration of follow up

\begin{tabular}{|c|c|c|c|c|c|c|}
\hline $1-5$ years & 44 & 43 & $\begin{array}{l}1.451(0.706- \\
2.982)\end{array}$ & 0.312 & $\begin{array}{l}1.336(0.547 \\
-3.261)\end{array}$ & 0.525 \\
\hline 5-10years & 89 & 59 & $\begin{array}{l}1.859(0.880- \\
3.929)\end{array}$ & $0.104^{*}$ & $\begin{array}{l}1.181(0.610- \\
3.044)\end{array}$ & 0.450 \\
\hline Greater than 10 years & 35 & 43 & 1 & & 1 & \\
\hline
\end{tabular}

\section{Member of diabetic association}


Adherence

\begin{tabular}{lccllll}
\cline { 2 - 4 } Variable & Poor & Good & $\begin{array}{c}\mathrm{COR} \\
{[95 \% \mathrm{Cl}]}\end{array}$ & $\begin{array}{l}\text { P- } \\
\text { value }\end{array}$ & AOR [95\%Cl] & $\begin{array}{l}\text { P- } \\
\text { value }\end{array}$ \\
\hline Yes & 76 & 52 & 1 & & 1 & \\
\hline No & 92 & 93 & $\begin{array}{l}1.477(0.937- \\
2.330)\end{array}$ & $0.093^{*}$ & $\begin{array}{l}2.495(1.162- \\
5.360)\end{array}$ & $0.019 * *$ \\
& & & & &
\end{tabular}

\section{Missed dietary planning}

$\begin{array}{llllll}\text { Yes } & 164 & 94 & \begin{array}{l}0.045(0.016- \\ 0.128)\end{array} & 0.000 * & \begin{array}{l}0.026(0.008- \\ 0.089)\end{array}\end{array} \quad \begin{array}{ll}0.000 * * \\ \end{array}$

$\begin{array}{lllll}\text { No } & 4 & 51 & 1 & 1\end{array}$

\section{Coo morbidity}

\begin{tabular}{|c|c|c|c|}
\hline Yes & 53 & 59 & $\begin{array}{l}1.489(0.936- \\
2.368)\end{array}$ \\
\hline
\end{tabular}

$\begin{array}{lllll}\text { No } & 115 & 86 & 1 & 1\end{array}$

Note: * Significantly associated factors at $\mathrm{p}$ value $=0.25$, ** significantly associated at 0.05 : COR: Crude

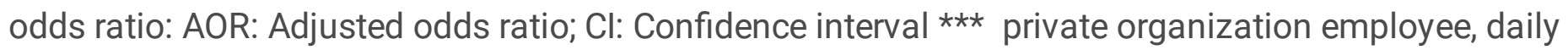
laborers and house wife.

\section{Discussion}

The overall magnitude of poor dietary adherence (non adherence) to dietary recommendation was 53.7\%among type II DM adult patients on follow-up at Nigist Elleni Mohammed Memorial Referral and Teaching Hospital, southern Ethiopia. The finding is in line with studies done in Adiss Ababa city and dedre birhan teaching Hospital that showed the magnitude of the poor adherence to dietary recommendation among adult type II DM patients was $51.4 \%$ and $55.7 \%$ respectively $[7,11]$. In contrary, findings of this study was lower than the study conducted in Mekelle, Felege hiwot hospital (Bhair Dar), Harari and Kenya where $69 \%, 64.1 \%, 60 \%$ and $59 \%$ of participants did not follow dietary recommendations respectively [13-16]. However the finding of this result was lower than the study done in Urban Area of Urmia, Northwest of Iran where $26.2 \%$ of participants had poor dietary adherence [17]. This might be due to difference in sample size, socioeconomic and cultural factors among different setups.

Respondents who had medium wealth status were over 3 times more likely to have poor dietary practice than those who had high wealth status. This result is in agreement with a study done in Diredawa referral hospital, Ethiopia where the study participants who had a medium monthly income were 2.33 times more likely to have poor adherence to diet [18]. It is also in line with a report from studies done in Addis Ababa Tikur Anbessa specialized hospital, Felege Hiwot Hospital, North west Ethiopia and Ayder referral 
hospital, Northern Ethiopia $[16,19]$. This might be because of those who have economic constraints cannot have enough money to buy different types of foods to fulfill their daily requirements.

Family and social support was another important factor affecting dietary practice of patients with type II DM. Similar results were reported from Addis Ababa, Ethiopia and Jimma medical college ,south west Ethiopia[16, 20] which showed there was a positive association between social support and adherence to lifestyle recommendations. This is due to the fact that when they couldn't get help from their nearby family they may feel despondent and then they might miss their dietary meal plan, including taking the excess sweetness and alcohol. Positive family support is the means of promoting preventive measures like good dietary practice and other diabetes self-care practices $[17,18]$. It is similar with studies done in Taiwan and Nekemte Referral Hospital, Ethiopia that family and social support was passively associated with good adherence of dietary practice among type II DM patients [21, 22].

Being a member of the Diabetic association was also significantly associated with adherence to dietary recommendation which was in line with the study in Felegehiwot Hopital, North west Ethiopia [19]. This might be due to the association's regular support given to the patients including support on self care in addition to securing medicine to some of the lower income members and blood glucose testing with a relatively lower price.

Assessing the level of dietary practice using self-reported dietary practice was one of the limitations of the study. Besides, using self-reported dietary adherence as a measure of the level of adherence may introduce social desirability or recall bias.

\section{Conclusion And Recommendation}

The overall magnitude of the poor adherence to dietary practice among type II diabetes mellitus adult patients was $53.7 \%$ which is not going in line with international recommendations of diabetic self care. Besides, having large family size, occupations, being low wealth status, lack of family support and not being member of diabetic association were the factors associated with poor adherence of dietary practice among type II DM patients. Ever concerned bodies should focus on addressing family planning issues, creating job opportunities and increasing household economic status of patients with type II DM. Lastly, support from the family members and expanding the diabetic association service are essential factors to promote dietary practice among type II DM adult patients.

\section{Declarations}

\section{Acknowledgment}

We would like to thank data collectors, respondents and all individuals for their contribution.

\section{Availability of data and material}


The datasets are available from the corresponding author on reasonable request.

\section{Authors' contribution}

MS Designed and supervised the study, analyzed and interpreted the data

DT Assisted the analysis and interpretation of the data

TT Prepared and submitted the final manuscript for publication

All authors read and approved the final manuscript.

\section{Financial disclosure}

There was no fund

\section{Competing interest}

The authors declare that they have no competing interests.

\section{Abbreviations}

AOR adjusted odds ratio

COR crude odds ratio

DM diabetes mellitus

NEMMTH Nigist Eleni Mohamed Memorial Teaching Hospital

\section{References}

1. ADA. Diagnosis and classification of diabetes. Diabetes Care. 2017; 40(Supplement 1):S11-24 http://doi.org/10.2337/dc17-S005

2. International diabetes federation. IDF diabetes atlas 9th edition. 2019 www.diabetesatlas.org

3. WHO. Diabetes Fact sheet. 2018 http://wwwwhoint/news-room/fact-sheets/detail/diabetes.

4. ADA. Nutritional Recommendation and Intervention for Diabetes. Diabetes Care. 2008; 31(1).

5. WHO. Double burden of malnutrition. 2015 http://wwwwhoint/nutrition/double-burden-malnutrition.

6. CDC. National diabetic fact sheet: national estimates and general information on diabetes and prediabetes in the United States, 2011. Atlanta, GA: U.S Department of Health and Human Services.

7. Abebe et al. Dietary practice and associated factors among type 2 diabetic patients: a cross sectional hospital based study, Addis Ababa, Ethiopia. Springer plus. 2015;4(15). 
8. Gill GV, Mbanya, J.C., Ramaiya, K.L. and Tesfaye, S. A sub-Saharan African perspective of diabetes. Springer nature diabetologia. 2009; 52: 8-16.

9. NEMMTH. Hospital's annual report. 2019.

10. Ayele et al. Level of adherence to dietary recommendations and barriers among type 2 diabetic patients. a cross sectional study in an Ethiopian Hospital. Clin diabetes Endocrinol. 2018 https://doiorg/101186/s40842-018-0070-7.

11. Mohammed et al. Adherence to dietary recommendation and associated factors among diabetic patients in Ethiopian teaching hospitals. Pan African Medical Journal.2019;33:260

12. Rowley C. Factors influencing treatment adherence in diabetes. 2008.

13. William kiberenge maina et al. Knowledge, attitude, and practices related to diabetes among community members in four provinces in Kenya. A cross sectional study. PAMJ.2010 ISSN 19378688

14. Ayele K,Tesfa T, Girma E. Self care behavior among patients with Diabetes in Harari, Eastern Ethiopia: The health belief model perspective. Plos One 2012;7(4).

15. YM Demilew. Dietary practice and associated factors among type 2 diabetic patients in Felege Hiwot Regional Referral Hospital, Bahir Dar, Ethiopia. BMC. 2018;11:434.

16. Berhe KK et al. Adherence to Diabetes self-management practices among Type 2 Diabetic Patients in Ethiopia; A cross sectional study. Greener journal of medical sciences. 2012;5(98):211-21.

17. Zahra Yekta et al. Assessment of Self Care Practice and Its Associated Factors among Diabetic Patients in Urban Area of Urmia, Northwest of Iran. J Res Health Sci. 2011; 11(1):33-8.

18. Mohammed AS AF, Tadiwos $Y$, Woldekidan NA. Level of adherence to the dietary recommendation and glycemic control among patients with Type 2 Diabetes Mellitus in Diredawa referral hospital, Ethiopia. 2020-13:2605-12.

19. Solomon A Feleke et al. Assessment of the level and associated factors with knowledge and practice of Diabetes Mellitus among Diabetic Patients Attending at FelegeHiwot Hospital, NSorthwest Ethiopia. Clinical Medicine Research. 2013;2(6):110-20.

20. Hailu E,Mariam WH, Belachew T, Birhanu Z. Self-care Practice and Glycemic Control Among Adults With Diabetes at Jimma University Specialized Hospitals in south -west Ethiopia: a cross sectional study. Afr J Prm Health care Fam Med. 2012;4(1).

21. Amante T et al. Self Care Practice And Its Predictors Among Adults With Diabetes Mellitus On Follow Up At Nekemte Hospital Diabetic Clinic, West Ethiopia. World Journal of Medicine and Medical Science. 2014;2(3):1-16.

22. Wu SF, Courtney M, Edwards H, Mc Dowel J, Shortridge-Bagget LM, Chang PJ. Self-efficacy, outcome expectations and self-care behavior in people with type 2 diabetes in Taiwan. J Clin Nurs. 2007; 16(11c):250-7. 